Résumés des conférences et travaux

\title{
Histoire de l'Extrême-Orient prémoderne et épigraphie chinoise
}

\section{Pierre Marsone}

\section{OpenEdition}

\section{Journals}

Édition électronique

URL : https://journals.openedition.org/ashp/341

DOI : 10.4000/ashp.341

ISSN : 1969-6310

Éditeur

Publications de l'École Pratique des Hautes Études

\section{Édition imprimée}

Date de publication : 1 octobre 2008

Pagination : 342-345

ISSN : 0766-0677

\section{Référence électronique}

Pierre Marsone, "Histoire de l'Extrême-Orient prémoderne et épigraphie chinoise », Annuaire de l'École pratique des hautes études (EPHE), Section des sciences historiques et philologiques [En ligne], 139 | 2008, mis en ligne le 07 janvier 2009, consulté le 12 juillet 2021. URL : http://journals.openedition.org/ashp/ 341 ; DOl : https://doi.org/10.4000/ashp.341 


\title{
HISTOIRE DE L'EXTREAME-ORIENT PRÉMODERNE ET ÉPIGRAPHIE CHINOISE
}

\author{
Maître de conférences : M. Pierre Marsone
}

Programme de l'année 2006-2007 : I. Histoire des empires sinisés (Liao, Jin) et de la Chine sous les Mongols : la formation de l'empire Khitan. - II. Initiation à l'épigraphie chinoise : stèles funéraires de dignitaires ouighours à la cour mongole (Yuan).

\section{Histoire des empires sinisés (Liao, Jin) et de la Chine sous les Mongols : la formation de l'empire Khitan}

Le volet historique des conférences de cette année a entrepris l'étude de l'origine et de la formation de l'empire des Khitan, plus connu en chinois sous le nom de dynastie Liao (907-1125). Cette dynastie de souverains non han qui recouvrit une partie limitée de la Chine han, occupe dans l'histoire de ce pays une place marginale, en dépit de son importance dans l'histoire de l'Eurasie. De fait, les Chinois connaissent au mieux le nom de cette dynastie dont dépendit pourtant l'actuelle ville de Pékin pendant plus de deux ans. Or les Khitan ne sont pas un peuple barbare surgi de la steppe en 907 par quelque hasard de l'histoire. Ce sont même, pourrait-on dire, de « vieilles connaissances » des souverains chinois qui entretenaient avec l'empire du Milieu des relations mouvementées depuis plus de cinq cents ans. L'étude approfondie de l'empire Khitan, à laquelle se destine, à long terme, le présent séminaire, se doit donc de commencer par l'examen de ces quelques siècles de contacts faits de ralliements, de guerres et de répressions suivies de nouveaux ralliements et de l'octroi régulier, aux chefs khitan, de dignités et de fonctions officielles. On saisit alors mieux que les « barbares » qui font irruption dans la chronologie chinoise sont un peuple certes nomade, mais dont l'aristocratie n'est pas complètement étrangère au système administratif, rituel et culturel chinois.

Les annales dynastiques chinoises, principal matériau de la partie historique du séminaire, fournissent à ce sujet des informations partielles, très incomplètes et dispersées, mais dont la réunion permet d'esquisser une véritable histoire politique, accompagnée d'une description ethnologique sommaire, du peuple khitan avant la création de son empire. Ce travail de collation et d'analyse des matériaux historiques n'a jamais été entrepris sérieusement. Cette étude se révèle pourtant très instructive sur la façon dont la Chine a géré de façon constante ses relations avec les peuples voisins, et particulièrement ces tribus du Nord qui, à travers toute l'histoire chinoise, ont constitué pour l'empire la première menace militaire. Le séminaire de cette année a été consacré à la première partie de projet : l'histoire des Khitan entre le moment de l'apparition de leur entité ethnique au Iv siècle jusqu'à la fin de la dynastie Sui (618). Le nom des Khitan apparaît pour la première fois dans la chronologie du Livre des Wei (Weishu, achevé en 554) et dans le court paragraphe monographique que ces annales lui consa- 
crent. Selon cette monographie, le pays des Khitans se trouve à l'est de celui des Kumo $\mathrm{Xi}$. Selon des notions ethnologiques très approximatives, les deux peuples sont décrits comme étant « du même genre mais d'espèce différente » (yi zhong tong lei). Mais c'est l'Histoire du Nord (Beishi, achevée en 659), qui donne la plus ancienne indication sur les Khitan, plaçant leur entrée dans l'histoire chinoise en 345, lors de leur défaite et de celle des Kumo Xi devant un Xianbei, Murong Huang (297-348), fondateur de l'État des Yan antérieurs (335-385). À ce moment, les survivants des Kumo Xi semblent avoir rejoint les Khitan dans la région de Songmo, une région aux contours mal définis, située au nord de l'actuelle ville de Chifeng en Mongolie-Intérieure et incluant le bassin de la rivière Laohahe. Ces tribus durent cependant continuer à opérer des razzias chez leurs voisins puisque quelques décennies plus tard, en 388, Tuoba Gui des Wei du Nord (385-534) et Murong Chui des Yan postérieurs (384-407) leur infligèrent une nouvelle défaite. À partir de 407, les Yan du Nord (407-436) joignirent à la politique de répression une politique conciliatrice. Après une nouvelle répression, ils s'attachèrent les chef khitan qui se rendaient en leur donnant le titre de « rois ralliés au bien » (guishanwang), et ouvrirent à Yingqiu, dans la région de l'actuelle Chaoyang, un marché frontalier. C'est à partir de ce moment que des Khitans commencèrent à apporter des " produits de leur terroir » (fangwu) à une dynastie chinoise, mais le premier tribut du pays des Khitan n'est attesté qu'en 437. À partir de cette date, le tribut des Khitan, composé de chevaux et de fourrures, sera régulier. En 466, le chef (mofu) khitan Hehechen est si vivement impressionné par la cour des Wei que, lorsqu'il rentre dans son pays, il convainc les chefs de nombreuses autres tribus de se rallier aux Wei. En 479, le royaume de Koguryo s'entend secrètement avec les Rouran, menaçant directement les Khitan. Inquiet devant les massacres qui s'annoncent, le chef khitan, Hewuyu vient avec trois mille chars et dix mille personnes chercher la protection des Wei. L'empereur les laisse s'installer à l'est de la rivière Bailangshui, actuelle Dalinghe. À l'époque du roi des Rouran Yujiulü Chounu (508-520) et de son frère Anagui, les Khitans entrèrent probablement dans la sphère d'influence de ses derniers, sans pour autant rompre leur relation avec l'empire des Wei. À partir de 552, les Khitan, émancipés des Rouran vaincus par les Turks (Tujue), entretiennent avec la Chine des Qi du Nord des relations plus complexes, les harcelant par leurs incursions et leurs razzias tout en continuant dans le même temps de leur apporter le tribut. Après plusieurs campagnes de répression, celle de 605, menée conjointement par la Chine et les Tujue, porte aux Khitan un coup décisif : quarante mille Khitan sont capturés, les hommes exécutés, femmes, enfants et bétail donnés aux Tujue. La leçon est si rude qu'à partir de 611 les Khitans qui ont échappé au massacre feront partie de la coalition des Sui et des Tujue qui attaqua la Corée trois ans de suite.

La lecture des sources historiques a aussi été l'occasion de traduire la description ethnographique des Khitan, très sommaire, consignée dans les annales. Celle-ci nous apprend seulement que, chez les Khitan, pleurer à la mort des parents est considéré comme un signe de faiblesse et que les cadavres sont simplement placés au sommet des arbres, dans les montagnes. Trois ans plus tard, on récupère les ossements et on les incinère, puis on trinque en formulant un vœu énigmatique : «Pendant les mois d'hiver, que je mange tourné vers le soleil, quand je chasse, que je prenne beaucoup de sangliers et de cerfs. » Ces maigres informations consacrées spécifiquement aux Khi- 
tan doivent être complétées par celles qui sont données à propos des tribus voisines, dont les sources nous disent parfois que, sur tel ou tel point, elles sont semblables aux Khitan. Nous avons donc, dans le cadre séminaire, traduit et commenté les informations qui nous sont données au sujet des peuples voisins des Khitan à cette période : les Wuji (Malgal), Xi, Shiwei, Doumolou, Didouyu et Wuluohou. Les travaux de l'année prochaine, qui porteront sur les relations entre les Khitan et l'empire chinois des Tang nous permettront de prendre toute la mesure de l'ancienneté et de l'intensité des relations entre la Chine des Tang et les Khitan.

\section{Initiation à l'épigraphie chinoise : stèles funéraires de dignitaires ouighours à la cour mongole (Yuan)}

La partie épigraphique des conférences nous a permis d'étudier, par le biais du texte d'une stèle, la vie et la carrière d'un dignitaire ouighour à la cour mongole des Yuan (1260-1368) Alghun Shari (Aluhun sali, 1245-1307). Le texte fut écrit par le célèbre lettré et calligraphe Zhao Mengfu (surnommé Songxue daoren, 1254-1322), un proche collaborateur de Alghun Shali à la tête de l'État. Le texte commence par rappeler qu'en vertu du fait que les Ouighours de Gaochang (act. Turfan au Xinjiang) furent le premier peuple sédentaire à se rallier à Gengis khan, leur roi (l'iduq qut) fut considéré par le Conquérant comme son cinquième fils, et ses descendants étaient mis au rang de princes impériaux.

Alghun Shali, originaire de Beshbaliq, capitale septentrionale et principale du royaume Ouighour, était issu d'une famille de fonctionnaires depuis plusieurs générations. Son père, Qitai Sali, avait reçu une solide formation bouddhique. Sa mère, madame Zhang, était chinoise, comme sa grand-mère paternelle. Alghun Shali étudia aussi le bouddhisme. Il fut formé par le maître national 'Phags-pa (1235-1280), le grand conseiller tibétain de l'empereur Qubilai. La stèle dit qu'en quelques mois « il apprit l'écriture [de 'Phags-pa], les langues de tous les pays voisins et le chinois. Constatant combien il était doué, Qubilai lui fit étudier l'écriture du chinois. En peu de temps il connut les classiques, les histoires et les cent écoles. Quant au yin/yang, au comput du calendrier, aux diagrammes et aux apocryphes ainsi qu'aux techniques, il n'y eut rien qu'il ne connût parfaitement. » Si cette citation peut comporter une part d'exagération, elle signifie bien qu'Alghun Shali dut recevoir une formation confucéenne et technique suffisante à l'accomplissement des hautes tâches de gouvernement auxquelles il fut appelé. De fait, Alghun Shali faisait partie des lettrés proches de l'empereur à quarante ans et connut par la suite une carrière aussi régulière que brillante : en 1284, il fut nommé Grand maître du protocole de cour (Chaolie dafu), assistant impérial de gauche (zuo shiyi fengyu). Refusant une nomination supplémentaire comme directeur du Conservatoire des sages (Jixianguan) qui supervisait le taoïsme il fut nommé Grand maître de l'harmonie au palais (Zhongshun dafu) et membre du Conservatoire des sages et de l'Institut d'astrologie (Taishiyuan) qui compilait les annales historiques. Dans une ascension éclair, il fut nommé dès 1287 Grand mâ̂tre de la félicité glorieuse (Ronglu dafu, titre de rang 1b), chargé des affaires gouvernementales (pingzhang zhengshi). En 1291, probablement atteint par les conséquences de la chute et de l'exécution de Sangha, un ministre musulman ouighour ou tibétain 
très corrompu, Alghun Shali, quoiqu'il se fût toujours opposé à ce dernier, se retira des fonctions de gouvernement. Mais dès 1293 il devint directeur de l'Institut d'astrologie et, après la mort de Qubilai (1294), à la demande de l'impératrice douairière Yusheng, il recommença à accomplir des tâches de gouvernement pour Chengzong qui mit en lui une confiance totale, Alghun Shali joua alors un rôle crucial dans le recrutement et le choix des lettrés de la cour. C'est suite au décès de Chengzong qu'Alghun Shali, profondément affecté, serait tombé malade, et qu'il mourut.

Outre la biographie complète et les faits qu'elle rapporte, et qui ne pourraient être connus par aucune autre source, cette stèle donne quelques précisions sur les détails de l'administration mongole à la tête de la Chine sous la dynastie Yuan. Plus encore, à travers l'exemple d'un Ouighour, elle manifeste le rôle capital que jouaient à la cour mongole les semuren, ces ressortissants de peuples non mongols mais alliés aux Mongols, qui constituaient sous les Yuan la deuxième classe sociale, juste au-dessous des Mongols mais au-dessus des Chinois. 